\title{
STABILITY ANALYSIS OF THERMO-VISCO-PLASTIC MATERIALS UNDERGOING HIGH-RATE SHEAR DEFORMATIONS
}

BY

REZA MALEK-MADANI (Dept. of Mathematics, U. S. Naval Academy, Annapolis, MD)

AND

RAOUF ALI RAOUF (Dept. of Mechanical Engineering, U. S. Naval Academy, Annapolis, MD)

Abstract. In this paper we present an energy estimate for two systems of partial differential equations that govern thermo-mechanical behavior of materials undergoing simple and anti-plane shear deformations. A linearized stability analysis is then carried out for the case of simple shear deformation with an exponentially softening stress-strain law. A series of numerical experiments on the fully nonlinear one-dimensional system of partial differential equations constitutes the final section of the paper.

1. Introduction. This paper studies certain special solutions of a system of partial differential equations associated with high-rate shearing of thermo-visco-plastic solids. The dynamic behavior of the variables $v$ and $\theta$ is governed by the following system of equations:

$$
\begin{array}{ll}
v_{t}=\operatorname{div} \sigma & \text { conservation of linear momentum, } \\
\theta_{t}=\lambda \Delta \theta+\sigma \cdot \nabla v & \text { energy balance, }
\end{array}
$$

where the divergence and gradient operations are with respect to the spatial coordinates $x$ and $y, v$ is the velocity perpendicular to the $(x, y)$-plane, $\theta$ is the temperature, $\lambda$ is a measure of thermal conductivity of the material, and $\sigma$ contains the appropriate components of the Cauchy stress tensor. This paper studies two forms of system (1):

1. Simple shear condition: This restricts Eqs. (1) to one spatial dimension $x$. The reduced system is

$$
v_{t}=\sigma_{x}, \quad \theta_{t}=\lambda \theta_{x x}+\sigma v_{x}
$$

where $v=v(x, t)$ is the velocity perpendicular to the $x$-direction, and $\sigma=\sigma(x, t)$ contains only one component.

Received June 6, 1996.

1991 Mathematics Subject Classification. Primary 73B30, 73E99.

Note added in proof. This paper is dedicated to the memory of the second author, Raouf Ali Raouf, who passed away on 19 April, 1997. 
2. Anti-plane shear: This deformation is governed by the full form of Eqs. (1) where $v=v(x, y, t)$ and $\sigma=\sigma(x, y, t)$ is a vector consisting of the two nonzero shear components of the Cauchy stress.

The system of equations in (1) and (2) is closed once we specify a constitutive relation that represents the response of the material to the motions it undergoes. In this paper we use the following constitutive law:

$$
\sigma=\mathbf{f}(\theta, \nabla v)
$$

for anti-plane shear. For the case of simple shear, the constitutive law reduces to

$$
\sigma=f\left(\theta, v_{x}\right) .
$$

In (4) and (3) the functions $f$ and $\mathbf{f}$ are generally nonlinear functions of their arguments. Because the shear stresses in (4) and (3) depend on strain-rate $\left(v_{x}\right.$ and $\left.\nabla v\right)$, the materials that we are modeling may be interpreted as fluids. However, these models are used for solids that undergo severe plastic deformations at high strain rates (see Maddocks and Malek-Madani [1] for examples and references). In such a setting, (1)-(3) and (4) constitute a simple mathematical model of deformation for thermo-visco-plastic solids. Additionally, in the case of (4), when the constitutive function $f$ has the property that

$$
f(\theta, 0)>0
$$

i.e., when there is a residual stress present in the material in the absence of any strainrate, Eqs. (2) and (4) model material behavior described as generalized Bingham Plasticity (cf. Reiner [2]).

Typically the constitutive laws (3) and (4) satisfy some mild growth hypotheses. We will assume that the material softens with increasing temperature under the action of the deformation so that, in the case of simple shear,

$$
\frac{\partial f}{\partial \theta} \leq 0
$$

while it hardens with increasing strain-rate, i.e.,

$$
\frac{\partial f}{\partial v_{x}} \geq 0
$$

Equivalent constraints hold for the constitutive model in the anti-plane shear problem.

The bulk of this paper is concerned with the simple shear equations (2). These equations have recently been studied extensively because of their connection to modeling of shear localization and formation of shear bands. Tzavaras [3] demonstrated finite time blow-up of solutions to Eqs. (2) when $\lambda=0$ (i.e., when the process is adiabatic) and the stress-strain law is a power law $\sigma=\theta^{-\nu} u_{x}^{m} v_{x}^{p}$, where $u$ is the displacement. Jiang [5] proved the global existence of solutions to an initial-boundary value of Eqs. (2) when the stress-strain law is a restricted power law and when $\lambda>0$.

In this paper we prove an apriori bound for the solutions to an appropriate initialboundary problem for both systems (1) and (2), in the case where the stress-strain has a special exponential form, and when $\lambda \neq 0$. In addition, we give a stability criterion, based on a linearization argument, that demonstrates that the homogeneous solution to Eqs. (2) with velocity boundary and insulated thermal conditions remains stable for 
small perturbations. Finally, we present a series of numerical experiments on the latter problem which illustrate the range of applicability of the stability criterion.

2. Blow-up phenomenon in simple shear models. This section presents an example of the blow-up phenomenon in simple shear when the thermal process is adiabatic. This behavior is typical in certain initial-boundary value problems involving Eqs. (2), as demonstrated in Tzavaras [3] when the stress-strain law is a power law $\sigma=\theta^{-\nu} u_{x}^{m} v_{x}^{n}$. The argument put forth in Tzavaras' paper applies to a quite general set of initial data. Here we give a simple example of how the blow-up phenomenon is present when the constitutive model is an exponential law.

Consider the system

$$
v_{t}=\sigma_{x}, \quad \theta_{t}=\lambda \theta_{x x}+\sigma v_{x}, \quad \sigma=e^{-2 \theta} v_{x}
$$

with the boundary condition

$$
\sigma(1, t)=1
$$

and the initial condition

$$
\theta(x, 0)=1
$$

Let $\lambda=0$ in (8). Evaluate $\theta_{t}=\sigma v_{x}$ at the boundary $x=1$ and use the boundary condition (9) to get

$$
\frac{d \theta(1, t)}{d t}=v_{x}(1, t)
$$

Let $w(t) \equiv \theta(1, t)$. From (8c) we deduce that $v_{x}(1, t)=e^{2 w}$. Returning to (11), we see that the function $w$ satisfies the ordinary differential equation

$$
\frac{d w}{d t}=e^{2 w}, \quad w(0)=1
$$

where the initial condition in (12) follows from (10). It is easy to see from (12) that

$$
w(t)=\frac{1}{2} \log \frac{1}{\frac{1}{e^{2}}-t} .
$$

The above equation states that $w$ (i.e., the temperature at the surface) blows up when $t$ reaches $\frac{1}{e^{2}}$.

The above initial-boundary data is designed to uncouple the dynamic behavior of the temperature on the boundary from the rest of the material. Although one does not expect this uncoupling to be typical for solutions of the simple shear problem, we feel the phenomenon of blow-up of the temperature at some finite time is typical for these equations.

The above example was in the adiabatic setting. In the next section we derive an energy estimate for the non-adiabatic case. The estimate will curtail the growth of temperature, but does not rule out blow-up entirely. 
3. An energy estimate for simple shear. Consider Eqs. (2) with velocity boundary conditions

$$
v(0, t)=0, \quad v(1, t)=v_{0},
$$

and either Dirichlet or Neumann boundary conditions in temperature:

$$
\theta(0, t)=\theta(1, t)=0
$$

or

$$
\theta_{x}(0, t)=\theta_{x}(1, t)=0 .
$$

Theorem 1. Let $\sigma\left(\theta, v_{x}\right)$ be of the form

$$
\sigma\left(\theta, v_{x}\right)=e^{-\alpha \theta} f\left(e^{-\alpha \theta} v_{x}\right)
$$

where $\alpha>0$ and $f$ is a continuously differentiable function. Let $F$ be an anti-derivative of $f$. Let $(v(x, t), \theta(x, t))$ be a solution to Eqs. (2) satisfying the velocity boundary condition (14) and either Dirichlet or Neumann thermal boundary conditions. Let $(0, T)$ be the life span of the solution. Then the following identity holds:

$$
\left.\int_{0}^{1}\left[F\left(e^{-\alpha \theta} v_{x}\right)+\frac{\alpha \lambda}{2} \theta_{x}^{2}\right]\right|_{t=T} d x+\int_{0}^{T} \int_{0}^{1}\left[v_{t}^{2}+\alpha \theta_{t}^{2}\right] d x d t=\text { const },
$$

where the constant depends only on the initial data.

Proof. Multiply the first equation in (2) by $v_{t}$ and the second by $\alpha \theta_{t}$, integrate each equation with respect to $x$ from 0 to 1 and add the results:

$$
\int_{0}^{t}\left(v_{t}^{2}+\alpha \theta_{t}^{2}\right) d x=\int_{0}^{1}\left[\left(e^{-\alpha \theta} f\left(e^{-\alpha \theta} v_{x}\right)\right)_{x} v_{t}+\alpha \lambda \theta_{x x} \theta_{t}+\alpha e^{-\alpha \theta} f\left(e^{-\alpha \theta} v_{x}\right) v_{x} \theta_{t}\right] d x
$$

Next, integrate the first two terms on the right side of Eq. (19) by parts. This reduces (19) to

$$
\int_{0}^{1}\left(v_{t}^{2}+\alpha \theta_{t}^{2}+\frac{\alpha \lambda}{2} \frac{d}{d t} \theta_{x}^{2}\right) d x=-\int_{0}^{1} e^{-\alpha \theta} f\left(e^{-\alpha \theta} v_{x}\right)\left[v_{x t}-\alpha \theta_{t}\right] d x,
$$

where the contributions from the boundary vanish because of (14) and the fact that either $\theta_{x}$ or $\theta$ is zero on the boundary when either Neumann or Dirichlet thermal boundary data are specified. The integrand in the second integral in (20) can be expressed as an exact time derivative:

$$
e^{-\alpha \theta} f\left(e^{-\alpha \theta} v_{x}\right)\left[v_{x t}-\alpha \theta_{t}\right]=\frac{d F\left(e^{-\alpha \theta} v_{x}\right)}{d t},
$$

where $F$ is an anti-derivative of $f$. Using this fact in (20) and integrating the result with respect to $t$ leads to Eq. (18). This completes the proof of the theorem.

As a consequence of Eq. (18) we obtain the following apriori bounds on the solution pair $(v(x, t), \theta(x, t))$ :

$$
\sup _{\left(0, T^{\prime}\right)}\left\|\theta_{x}\right\|_{2} \leq M, \quad\|\| v_{t} \mid\left\|_{2} \leq M, \quad\right\| \theta_{t} \|_{2} \leq M,
$$


where the bound $M$ depends on the initial data alone. In (22) $\|\cdot\|_{2}$ and \|\|$\cdot\|\|_{2}$ refer to the $L^{2}$ norm taken over $(0,1)$ and $(0,1) \times(0, T)$, respectively. When Dirichlet thermal boundary conditions are specified, the standard embedding theorem and (22) imply

$$
\sup _{(0, T) \times(0,1)}|\theta(x, t)| \leq \text { const . }
$$

When $f$ is a monotone increasing function with $f^{\prime}(p) \geq k>0$, its anti-derivative $F$ will have at least quadratic growth, i.e., $F(p) \geq k p^{2}$. Then

$$
k \int_{0}^{1} e^{-2 \alpha \theta} v_{x}^{2} \leq \int_{0}^{1} F\left(e^{-\alpha \theta} v_{x}\right) d x
$$

which in turn is uniformly bounded by Eq. (18). The apriori estimate (23) guarantees that $e^{-2 \alpha \theta}$ remains bounded away from zero, which in turn implies

$$
\sup _{\left(0, T^{\prime}\right)}\left\|v_{x}\right\|_{2} \leq M .
$$

As in the case of the temperature, this estimate leads to the desired pointwise estimate on $v$. We have proved the following result.

Corollary 1. Let $(\theta, v)$ be a solution to the simple shear problem with $\sigma$ defined by (17), satisfying Dirichlet thermal boundary conditions. Suppose that the left side of (18) is finite when evaluated along the initial data $(\theta(x, 0), v(x, 0))$. Let $f$ in $(17)$ satisfy

$$
f^{\prime}(p) \geq k>0 \text {. }
$$

Then $v$ and $\theta$ are uniformly bounded in space and for all $t$ in the lifespan of the solution.

REMARK 1. The assumptions on the initial data and the constitutive function $f$ in the above corollary are rather mild. Consequently, we do not expect the finite time blow-up of the adiabatic case to be generic in the nonadiabatic problem when Dirichlet conditions are specified on the temperature.

REMARK 2. The special form of (17) was chosen in order to obtain (21). This form of the stress-strain law was first proposed by Maddocks and Malek-Madani [1] in connection with the stability analysis of the steady-state solutions of Eqs. (2).

4. An energy estimate for anti-plane shear. There is an analogue of Theorem 1 for the case of anti-plane shear.

Theorem 2. Let $\sigma_{1}$ and $\sigma_{2}$, the shear components of the Cauchy stress in (1), satisfy

$$
\sigma_{1}(\theta, \nabla v)=e^{-\alpha \theta} f_{1}\left(e^{-\alpha \theta} \frac{\partial v}{\partial x}\right), \quad \sigma_{2}(\theta, \nabla v)=e^{-\alpha \theta} f_{2}\left(e^{-\alpha \theta} \frac{\partial v}{\partial y}\right),
$$

with $f_{1}$ and $f_{2}$ arbitrary continuous functions of their argument and $\alpha>0$. Let $\mathbf{x}=$ $(x, y)$. Let $(v(\mathbf{x}, t), \theta(\mathbf{x}, t))$ be a solution to Eqs. (1) in the domain $D \times(0, T)$, satisfying Dirichlet boundary conditions in $v$ and either Dirichlet or Neumann boundary conditions in $\theta$ on the boundary $\partial D$. Then the following estimate holds:

$$
\int_{D}\left[F_{1}\left(e^{-\alpha \theta} v_{x}\right)+F_{2}\left(e^{-\alpha \theta} v_{y}\right)+\frac{\alpha \lambda}{2}|\nabla \theta|^{2}\right] d \mathbf{x}+\int_{0}^{T} \int_{D}\left(v_{t}^{2}+\alpha \theta_{t}^{2}\right) d \mathbf{x} d t=\text { const }
$$


with $F_{1}$ and $F_{2}$ anti-derivatives of $f_{1}$ and $f_{2}$ respectively, and the constant in (26) depending only on the initial conditions.

Proof. Multiply the first equation in (1) by $v_{t}$ and the second by $\alpha \theta_{t}$, integrate each equation with respect to $\mathbf{x}$ over $D$ and add the results:

$$
\int_{D}\left(v_{t}^{2}+\alpha \theta_{t}^{2}\right) d x=\int_{D}\left(v_{t} \operatorname{div} \sigma+\alpha \lambda \theta_{t} \Delta \theta+\alpha \theta_{t} \sigma \cdot \nabla v\right) d \mathbf{x} .
$$

Note that $v_{t} \operatorname{div} \sigma=\operatorname{div}\left(v_{t} \sigma\right)-\sigma \cdot \nabla v_{t}$. Using the divergence theorem and the fact that $v$ satisfies Dirichlet boundary conditions on $\partial D$ we see that

$$
\int_{D} v_{t} \operatorname{div} \sigma d \mathbf{x}=-\int_{D} \sigma \cdot \nabla v_{t} d \mathbf{x}
$$

An integration by parts on $\int_{D} \theta_{t} \Delta \theta d \mathbf{x}$ results in $-\frac{1}{2} \frac{d}{d t} \int_{D}|\nabla \theta|^{2} d \mathbf{x}$, much in the same spirit as the equivalent term in Theorem 1 . We end up with

$$
\int_{D}\left(v_{t}^{2}+\alpha \theta_{t}^{2}+\frac{\alpha \lambda}{2} \frac{d}{d t}|\nabla \theta|^{2}\right) d \mathbf{x}=-\int_{D}\left(\sigma \cdot\left(\nabla v_{t}-\alpha \theta_{t} \nabla v\right)\right) d \mathbf{x} .
$$

The integrand in the second integral in (29) can be expressed as an exact time derivative using the hypotheses (25):

$$
\frac{d F_{1}\left(e^{-\alpha \theta} v_{x}\right)}{d t}+\frac{d F_{2}\left(e^{-\alpha \theta} v_{y}\right)}{d t}=\sigma \cdot\left(\nabla v_{t}-\alpha \theta_{t} \nabla v\right)
$$

where $F_{1}$ and $F_{2}$ are anti-derivatives of $f_{1}$ and $f_{2}$. Using this fact in Eq. (29) and integrating the result with respect to $t$ leads to Eq. (26).

Unlike the simple shear case, the bounds on the gradient of temperature do not imply that the temperature is uniformly bounded even when Dirichlet boundary conditions are specified. It is hoped, however, that these bounds are fruitful in the numerical modeling of this problem in the future. In this connection see the recent paper by French and Garcia [4].

5. Linearized stability in the simple shear case. This section presents a linear stability result for the homogeneous solution of the simple shear equations

$$
v_{t}+\left(\mu(\theta) v_{x}\right)_{x}=0, \quad \theta_{t}=\lambda \theta_{x x}+\mu(\theta) v_{x}^{2},
$$

with boundary conditions

$$
v(0, t)=0, \quad v(1, t)=v_{0}, \quad \theta_{x}(0, t)=\theta_{x}(1, t)=0 .
$$

Here $\mu$ is a smooth function of temperature $\theta$ satisfying the conditions

$$
\mu>0, \quad \mu^{\prime}<0 .
$$

This system supports the homogeneous solution $(\bar{v}(x), \bar{\theta}(t))$ defined by

$$
\bar{v}(x)=v_{0} x,
$$

with $\bar{\theta}(t)$ satisfying the ordinary differential equation

$$
\theta_{t}=\mu(\theta) v_{0}^{2}
$$


In the special case

$$
\mu(\theta)=e^{-2 \alpha \theta}
$$

the function $\bar{\theta}$ is given by

$$
\bar{\theta}(t)=\frac{1}{2 \alpha} \ln \left(2 \alpha v_{0}^{2} t+c\right),
$$

where $c$ is a parameter. Since in this case the shear stress $\sigma$ is related to $v$ and $\theta$ by

$$
\sigma=e^{-2 \alpha \theta} v_{x}
$$

we see that the stress of the homogeneous solution decays with $t$ according to

$$
\bar{\sigma}(t)=\frac{v_{0}}{2 \alpha v_{0}^{2} t+c} .
$$

Our goal is to derive an estimate in terms of $\mu, \lambda$, and $v_{0}$ that would guarantee the boundedness (in an appropriate norm) of the perturbations of the homogeneous solution $(\bar{v}, \bar{\theta})$. To that end we consider the following forms for $v(x, t)$ and $\theta(x, t)$ and $\lambda$ :

$$
v(x, t)=v_{0} x+\varepsilon v_{1}(x, t)+\varepsilon^{2} v_{2}(x, t)+\cdots
$$

and

$$
\theta(x, t)=\bar{\theta}(t)+\varepsilon \theta_{1}(x, t)+\varepsilon^{2} \theta_{2}(x, t)+\cdots
$$

and

$$
\lambda=\lambda_{0}+\varepsilon \lambda_{1}+\cdots .
$$

We substitute Eqs. (38)-(40) into (31), differentiate with respect to $\varepsilon$ and set $\varepsilon$ equal to zero. This yields the following system of linear partial differential equations for the perturbations $v_{1}$ and $\theta_{1}$ :

$$
\begin{aligned}
v_{1, t}=\left(\mu(\bar{\theta}) v_{1, x}\right)_{x}+v_{0} \mu^{\prime}(\bar{\theta}) \theta_{1, x}, \quad \theta_{1, t} & =\mu^{\prime}(\bar{\theta}) v_{0}^{2} \theta_{1}+2 \mu(\bar{\theta}) v_{0} v_{1, x}+\lambda_{0} \theta_{1, x x} \\
v_{1}(0, t)=v_{1}(1, t) & =\theta_{1, x}(0, t)=\theta_{1, x}(1, t)=0 .
\end{aligned}
$$

We regard the homogeneous solution as stable if $v_{1}$ and $\theta_{1}$ are bounded for all times, and unstable otherwise. Equations (41) are linear differential equations with time-varying coefficients. Fortunately, this system is separable, for which we look for solutions $v_{1}(x, t)$ and $\theta_{1}(x, t)$ in the form

$$
v_{1}=\sum_{n=1}^{\infty} a_{n}(t) \sin n \pi x, \quad \theta_{1}=\sum_{n=0}^{\infty} b_{n}(t) \cos n \pi x .
$$

We substitute expansions (42) into (41) and collect coefficients of $\sin n \pi x$ and $\cos n \pi x$ : The order 0 terms yield

$$
b_{0}^{\prime}(t)=v_{0}^{2} \mu^{\prime}(\bar{\theta}(t)) b_{0}(t)
$$

while for $n \geq 1$ we obtain

$$
\begin{aligned}
& a_{n}^{\prime}(t)=-\pi^{2} n^{2} \mu(\bar{\theta}(t)) a_{n}-\pi n v_{0} \mu^{\prime}(\bar{\theta}(t)) b_{n} \\
& b_{n}^{\prime}(t)=2 \pi n v_{0} \mu(\bar{\theta}(t)) a_{n}+\left(-\pi^{2} n^{2} \lambda_{0}+\mu^{\prime}(\bar{\theta}(t)) v_{0}^{2}\right) b_{n}
\end{aligned}
$$


with $n=1,2, \ldots$. The system in (44) is equivalent to

$$
a_{n}^{\prime}=c_{1}(t) a_{n}+c_{2}(t) b_{n}, \quad b_{n}^{\prime}=c_{3}(t) a_{n}+c_{4}(t) b_{n},
$$

where

$$
\begin{array}{ll}
c_{1}(t)=-\pi^{2} n^{2} \mu(\bar{\theta}(t)), & c_{2}(t)=-\pi n v_{0} \mu^{\prime}(\bar{\theta}(t)), \\
c_{3}(t)=2 \pi n v_{0} \mu(\bar{\theta}(t)), & c_{4}(t)=-\pi^{2} n^{2} \lambda_{0}+\mu^{\prime}(\bar{\theta}(t)) v_{0}^{2} .
\end{array}
$$

The solution to Eq. (43) is given by

$$
b_{0}(t)=b_{0}(0) e^{-v_{0}^{2} \int_{0}^{t} \mu^{\prime}(\bar{\theta}(s)) d s} .
$$

In the special case of (35) and (36), the function $b_{0}$ takes the form

$$
b_{0}(t)=\frac{c b_{0}(0)}{2 \alpha v_{0}^{2} t+c} .
$$

In particular, $b_{0}$ decays to zero as $t \rightarrow \infty$.

The behavior of the solutions to Eqs. (45) is studied using Lyapunov's method. Let

$$
V_{n}=\frac{1}{2}\left(a_{n}^{2}+b_{n}^{2}\right)
$$

The derivative of $V_{n}$ along Eqs. (45) is

$$
\frac{d V_{n}}{d t}=c_{1} a_{n}^{2}+\left(c_{2}+c_{3}\right) a_{n} b_{n}+c_{4} b_{n}^{2} .
$$

The right side of Eq. (51) is a quadratic form. We define the matrix $A_{n}$ as the symmetric part of the matrix of coefficients in (45):

$$
A_{n}=\left[\begin{array}{cc}
c_{1}(t) & \frac{1}{2}\left(c_{2}(t)+c_{3}(t)\right) \\
\frac{1}{2}\left(c_{2}(t)+c_{3}(t)\right) & c_{4}(t)
\end{array}\right]
$$

Then (51) is equivalent to

$$
\frac{d V_{n}}{d t}=\left[\begin{array}{ll}
a_{n} & b_{n}
\end{array}\right] A_{n}\left[\begin{array}{l}
a_{n} \\
b_{n}
\end{array}\right] .
$$

We note that $A_{n}$ is real and symmetric. Let $\left\langle e_{1, n}(t), e_{2, n}(t)\right\rangle$ be the eigenvalues of $A_{n}$. Then, using a standard argument about quadratic forms, we arrive at

$$
\left[\begin{array}{ll}
a_{n} & b_{n}
\end{array}\right] A_{n}\left[\begin{array}{l}
a_{n} \\
b_{n}
\end{array}\right] \leq 2 \max \left(e_{1, n}(t), e_{2, n}(t)\right) V_{n} .
$$

Combining this estimate with (53), we have

$$
\frac{d V_{n}}{d t} \leq g_{n}(t) V_{n}
$$

where

$$
g_{n}(t)=2 \max \left(e_{1, n}(t), e_{2, n}(t)\right) .
$$

The differential inequality (55) implies that

$$
V_{n}(t) \leq V_{n}(0) e^{\int_{0}^{t} g_{n}(s) d s} .
$$


Now, the $L^{2}$ norm of the perturbations $v_{1}$ and $\theta_{1}$ are equal to

$$
\left\|v_{1}(t)\right\|^{2}=\frac{1}{2} \sum_{n=1}^{\infty}\left|a_{n}(t)\right|^{2}, \quad\left\|\theta_{1}(t)\right\|^{2}=\left|b_{0}(t)\right|^{2}+\frac{1}{2} \sum_{n=1}^{\infty}\left|b_{n}(t)\right|^{2} .
$$

Thus, recalling the definition of $V_{n}$ (see (50)), we have

$$
\left\|v_{1}(t)\right\|^{2}+\left\|\theta_{1}(t)\right\|^{2}=\left|b_{0}(t)\right|^{2}+\sum_{n=1}^{\infty} V_{n}(t)
$$

Next we use the estimates (48) and (57) in the above identity:

$$
\left\|v_{1}(t)\right\|^{2}+\left\|\theta_{1}(t)\right\|^{2} \leq\left|b_{0}(0)\right|^{2} e^{v_{0}^{2} \int_{0}^{t} \mu^{\prime}(\bar{\theta}(s)) d s}+\sum_{n=1}^{\infty} V_{n}(0) e^{\int_{0}^{t} g_{n}(s) d s} .
$$

The strategy for understanding the stability of the homogeneous solution then is to find conditions on $v_{0}$ and $\mu^{\prime}$ so that

$$
e^{v_{0}^{2} \int_{0}^{t} \mu^{\prime}(\bar{\theta}(s)) d s} \leq K, \quad e^{\int_{0}^{t} g_{n}(s) d s} \leq K, \quad \text { for all } t>0,
$$

for some positive constant $K$. It then follows from (60) that

$$
\left\|v_{1}(t)\right\|^{2}+\left\|\theta_{1}(t)\right\|^{2} \leq K\left(\left\|v_{1}(0)\right\|^{2}+\left\|\theta_{1}(0)\right\|^{2}\right),
$$

thus providing a measure of stability for the homogeneous solution.

We now prove (61) in the special case of (35). The matrix $A_{n}$ in this special case is

$$
A_{n}=\frac{1}{\left(e^{2 \alpha c}+2 \alpha t v_{0}^{2}\right)}\left[\begin{array}{cc}
-n^{2} \pi^{2} & (1+\alpha) n \pi v_{0} \\
(1+\alpha) n \pi v_{0} & -\lambda_{0} n^{2} \pi^{2}\left(e^{2 \alpha c}+2 \alpha t v_{0}^{2}\right)-\alpha v_{0}^{2}
\end{array}\right] .
$$

The eigenvalues of $A_{n}$ are:

$$
\lambda_{1,2}=\frac{-n^{2} \pi^{2}-\alpha v_{0}^{2}-\lambda_{0} n^{2} \pi^{2} h(t) \pm \sqrt{D}}{2 h(t)}
$$

where the discriminant $D$ is given by

$$
D=4(1+\alpha)^{2} n^{2} \pi^{2} v_{0}^{2}+\left(-n^{2} \pi^{2}+\alpha v_{0}^{2}+\lambda_{0} n^{2} \pi^{2} h(t)\right)^{2}
$$

and

$$
h(t)=e^{2 \alpha c}+2 \alpha v_{0}^{2} t .
$$

Note that $\lambda_{1} \geq \lambda_{2}$ and that $\lambda_{2}<0$ no matter what value the parameters take. Also, when we consider $\lambda_{1}$ as a function of $\left(\lambda_{0}, v_{0}, \alpha, c\right)$, i.e.,

$$
\lambda_{1}=\lambda_{1}\left(\lambda_{0}, v_{0}, \alpha, c\right)
$$

then

$$
\lambda_{1}(0,0, \alpha, 0)=0
$$

while

$$
\lambda_{1}\left(\lambda_{0}, 0, \alpha, 0\right)=-\lambda_{0} n^{2} \pi^{2} .
$$

Thus, when $\lambda_{0}>0, g_{n}$ in (56) contains a $-n^{2}$ term and the inequality in (61) is achieved by choosing $v_{0}$ small enough. In fact, we have the following lemma. 
Lemma 1. Suppose that

$$
v_{0}^{2}<\frac{\lambda_{0} \pi^{2}}{\alpha^{2}+\alpha+1} .
$$

Then the $L^{2}$ norm of the perturbations of the homogeneous solution of the special case (35) are bounded.

Proof. Note that the trace of the matrix $A_{n}$ in (63) is always negative. The determinant of $A_{n}$, on the other hand, is

$$
\frac{n^{2} \pi^{2}}{h(t)}\left(\lambda_{0} n^{2} \pi^{2} h(t)+\alpha v_{0}^{2}-(1+\alpha)^{2} v_{0}^{2}\right) .
$$

Hence, the determinant of $A_{n}$ is negative if

$$
v_{0}^{2}<\frac{\lambda_{0} n^{2} \pi^{2}\left(e^{2 \alpha c}+2 \alpha v_{0}^{2} t\right)}{\alpha^{2}+\alpha+1} .
$$

In the range of parameters of interest to us, the above expression takes on its minimum when $n=1, c=0$, and $t=0$. This minimum is precisely the right side of (70). Thus when $v_{0}$ is bounded by (70), the function $g_{n}$ in (61) is negative for all time and its integral for $t \in(0, \infty)$ is negative, so that we can choose $K=1$ in (62).

6. A numerical experiment. It is not known if the energy estimate (18) is sufficient to rule out blow-up in finite time of the solutions of the simple shear problem when Neumann thermal boundary conditions are specified. In this section we present a numerical study of this initial-boundary value problem that seems to point to an instability of the basic solution, and perhaps finite time blow-up, at least when the boundary data is large enough.

We consider the constitutive model

$$
\sigma=e^{-2 \alpha \theta} v_{x}
$$

where $\alpha>0$ is constant. For initial conditions we choose

$$
v(x, 0)=\frac{v_{0} x}{1+\varepsilon}\left(1+\varepsilon x^{2}\right), \quad \theta(x, 0)=0 .
$$

The boundary conditions are

$$
v(0, t)=0, \quad v(1, t)=v_{0}, \quad \theta_{x}(0, t)=\theta_{x}(1, t)=0 .
$$

As pointed out in the previous section, the pair of functions

$$
\bar{v}(x)=v_{0} x, \quad \bar{\theta}(x)=\frac{1}{2 \alpha} \ln \left(2 \alpha v_{0}^{2} t+c\right)
$$

form a basic solution of (73), (75), where $c$ is an arbitrary constant. According to Lemma 1 , we expect the pair $(\bar{v}, \bar{\theta})$ to be the asymptotic solution of $(73)-(75)$, at least when $v_{0}$ satisfies the bound (70) and $\varepsilon$ is small. As the numerical calculations below suggest, this basic solution seems to be stable when $\varepsilon$ and $v_{0}$ are small, while it seizes to explain the large time behavior of solutions of the initial-boundary value problem when $v_{0}$ or $\varepsilon$ is large. 


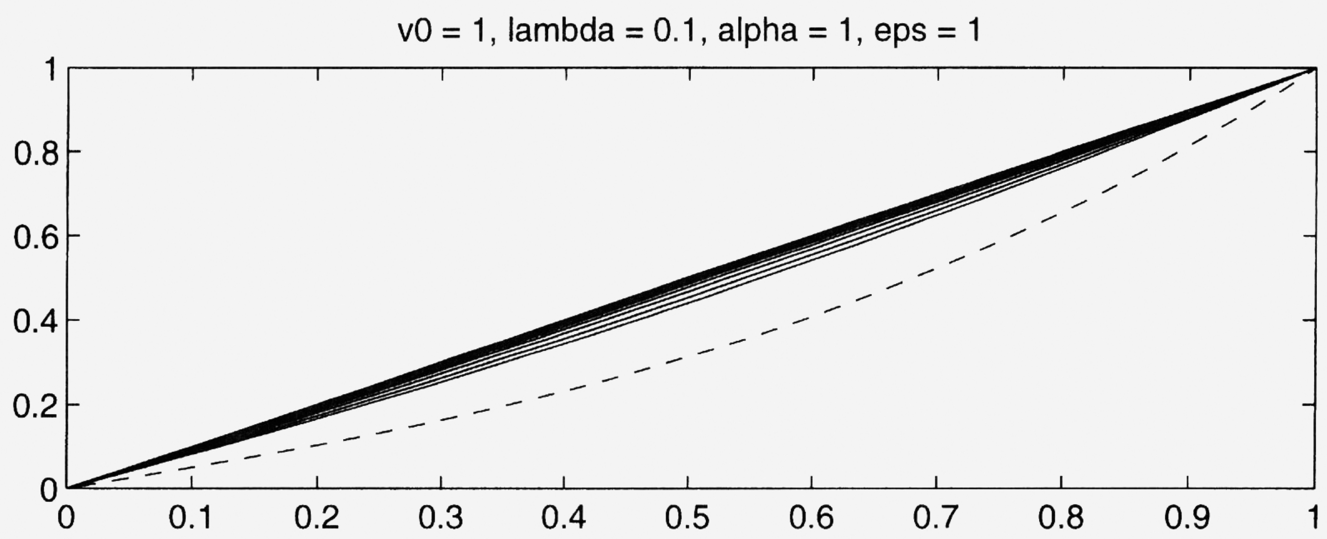

Temperature

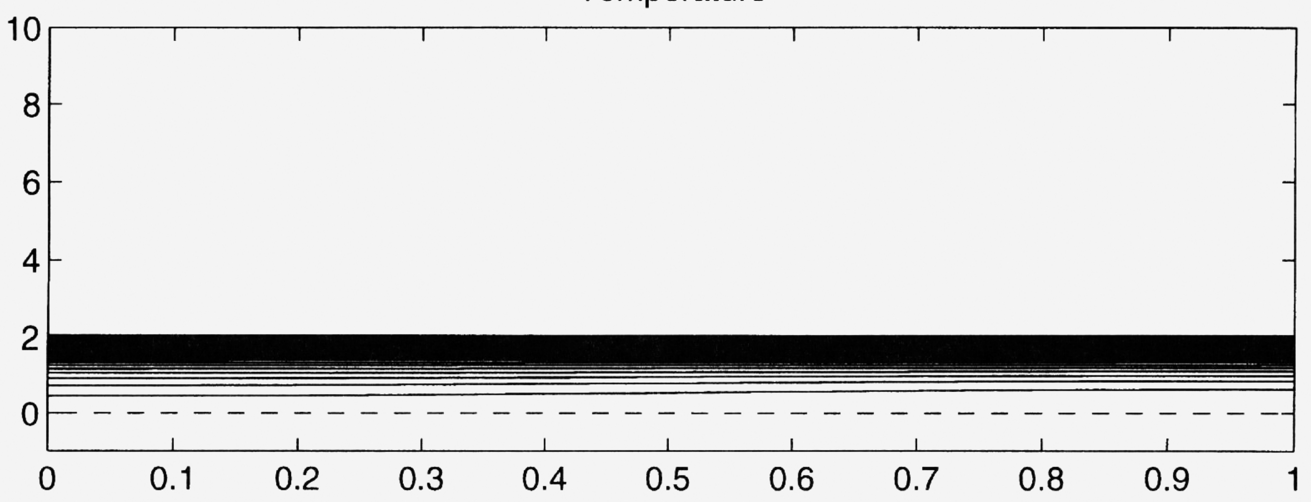

FIG. 1. Temperature and velocity fields for the simple shear problem with $v_{0}=1, \lambda=0.1, \alpha=1, \varepsilon=1$.

We employ the method of lines to numerically integrate

$$
v_{t}=\sigma_{x}, \quad \theta_{t}=\lambda \theta_{x x}+\sigma v_{x},
$$

with $\sigma$ given by (73). With a uniform mesh $\left\{x_{i}\right\}$ distributed along the $x$-axis, we discretize the right side of (77) using centered finite differences. Specifically, we discretize $\sigma_{x}$ by

$$
\frac{\sigma_{j+1 / 2}-\sigma_{j-1 / 2}}{h} \text {. }
$$

For the special case of (73), the term $\sigma_{j+1 / 2}$ is further discretized as

$$
\frac{1}{h}\left(\mu\left(\frac{\theta_{j+1}+\theta_{j}}{2}\right) \frac{v_{j+1}-v_{j}}{h}-\mu\left(\frac{\theta_{j}+\theta_{j-1}}{2}\right) \frac{v_{j}-v_{j-1}}{h}\right),
$$

where $\mu(\theta)=e^{-2 \alpha \theta}$. The terms $\theta_{x x}$ and $v_{x}$ in the energy balance equation are discretized using the standard centered difference schemes. In this way we are able to determine a numerical approximation to the solution of the initial value problem (77), (73)-(75), by computing the numerical solution of a rather large system of ordinary differential equations for the unkowns $\left(v_{j}(t), \theta_{j}(t)\right)$. 


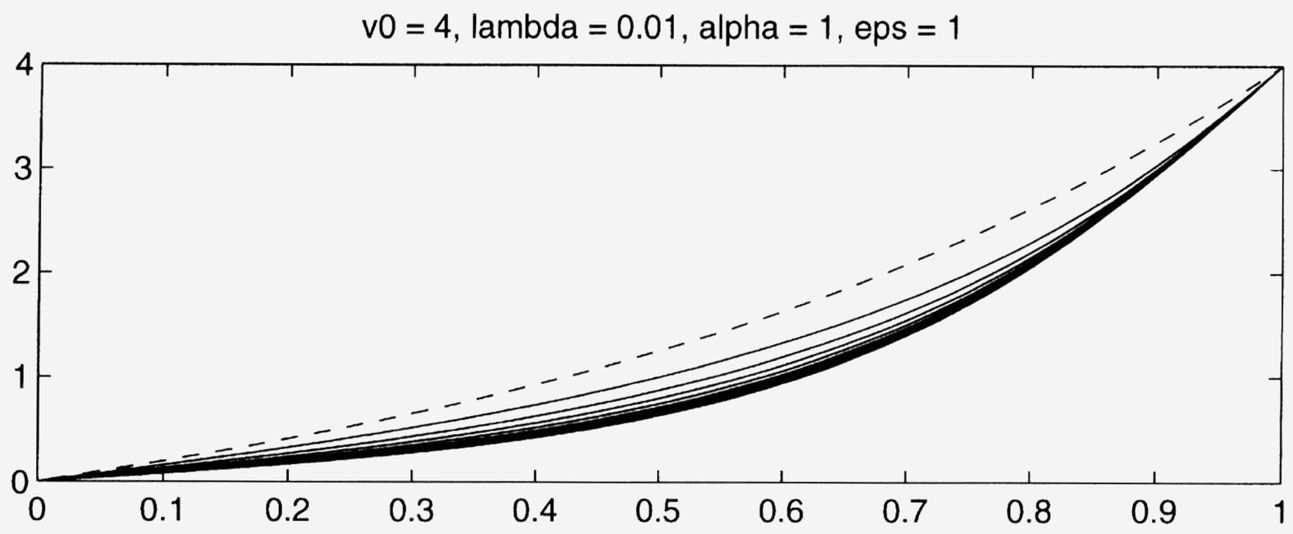

Temperature

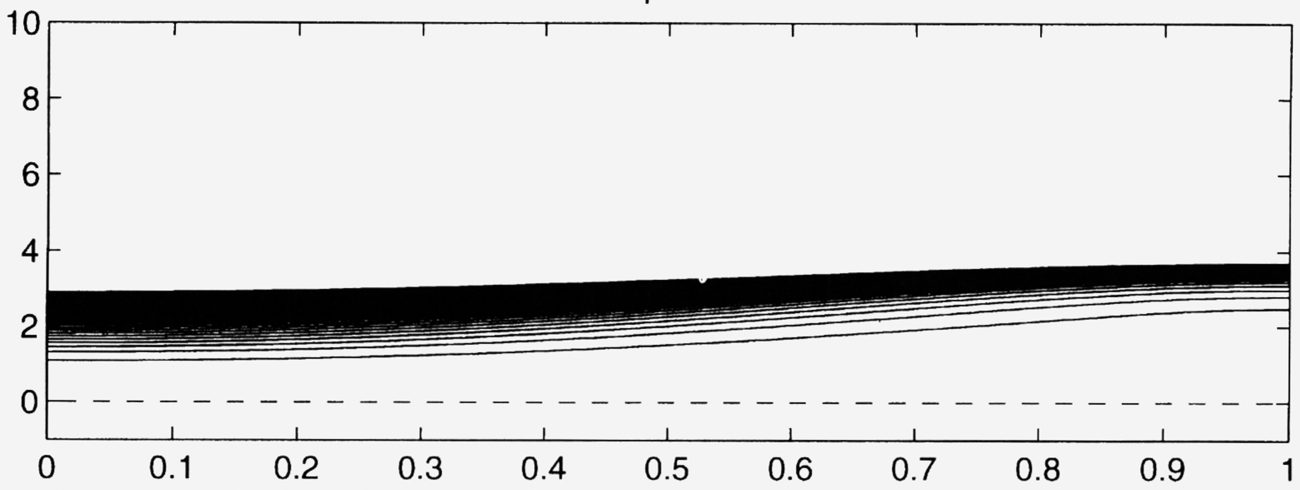

FIG. 2. Temperature and velocity fields for the simple shear problem with $v_{0}=4, \lambda=0.01, \alpha=1, \varepsilon=1$.

The resulting system of ordinary differential equations is then solved using Matlab's Runge-Kutta algorithm ode45. The listing of the Matlab M-files is included in the Appendix.

Each of the figures shows the evolution in time of the velocity and temperature fields for a particular set of parameter values. The horizontal axis is $x$, while the vertical axes are velocity and temperature, respectively. The dashed curves denote the initial profiles. Figure 1 shows the temperature and velocity fields when $v_{0}=1, \lambda=0.1, \alpha=1$, and $\varepsilon=1$. Even though the initial perturbation of the homogeneous shear is large, the solution seems to eventually converge to this state as time evolves. Figure 2 shows the temperature and velocity fields when $v_{0}=4, \lambda=0.01$, and $\alpha=\varepsilon=1$. Now the velocity field is actually moving away from the homogeneous shear velocity, while the temperature profile seems to be approaching a uniform state. Figure 3 is for the set of parameters $v_{0}=4, \lambda=10^{-5}$, and $\alpha=\varepsilon=1$. Near the boundary $x=1$, both the velocity and temperature are rather dramatically larger than the rest of the region, pointing to a localization of the shearing which becomes even more pronounced as the variable $t$ approaches infinity. 


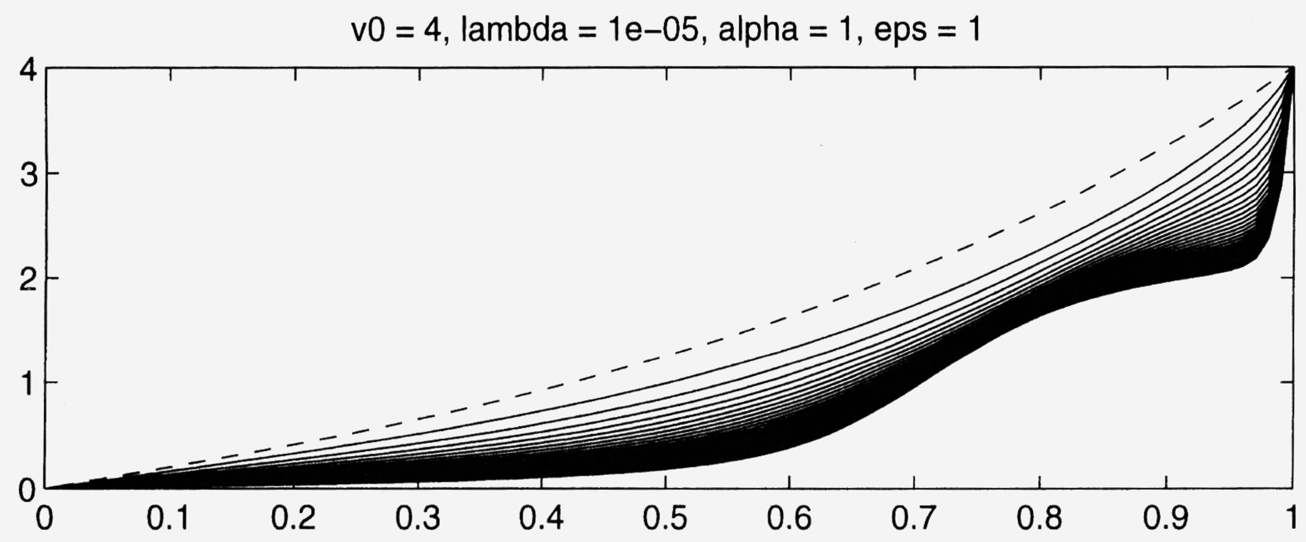

Temperature

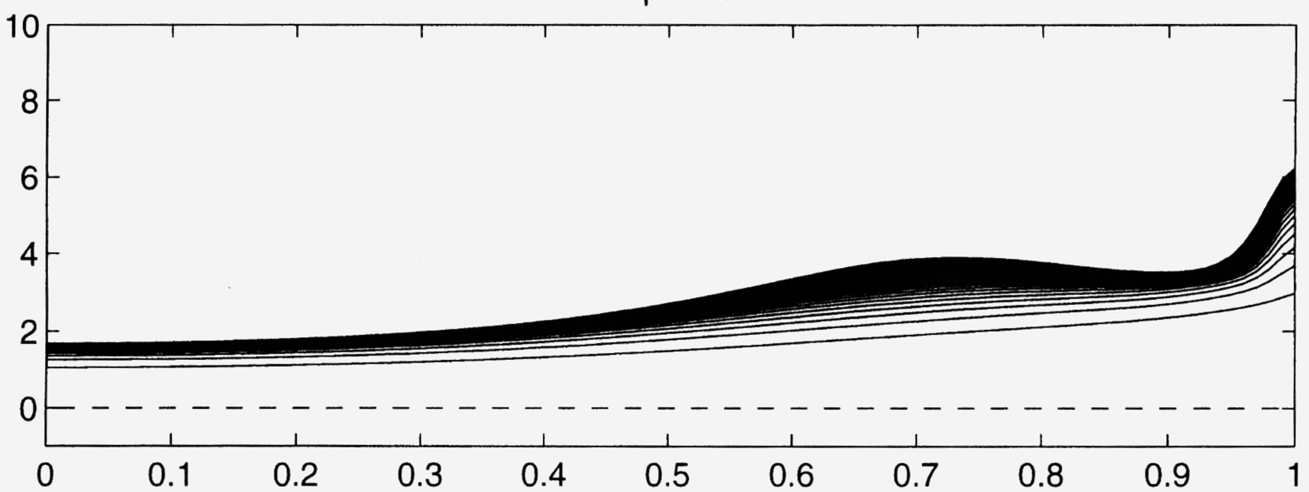

FIG. 3. Temperature and velocity fields for the simple shear problem with $v_{0}=4, \lambda=10^{-5}, \alpha=1, \varepsilon=1$.

Acknowledgment. The work of both authors was partially supported by the Office of Naval Research grant N0001496WR20011.

7. Appendix: Listing of the Matlab M-file. The following are the Matlab Mfiles that produced the numerical approximation and the graphs in Figure 3. The main program is as follows:

global lambda v0 enn h2 alpha;

$\%$

lambda=0.00001; alpha=1; eps = 1;

$\mathrm{v} 0=4 ;$ number_of_waves $=3000$; every_so_often $=100 ; \mathrm{dt}=0.01$;

count $=1$;

$\%$

enn=101;

$\%$

subscript $=(0:(e n n-1))$;

$\%$ 


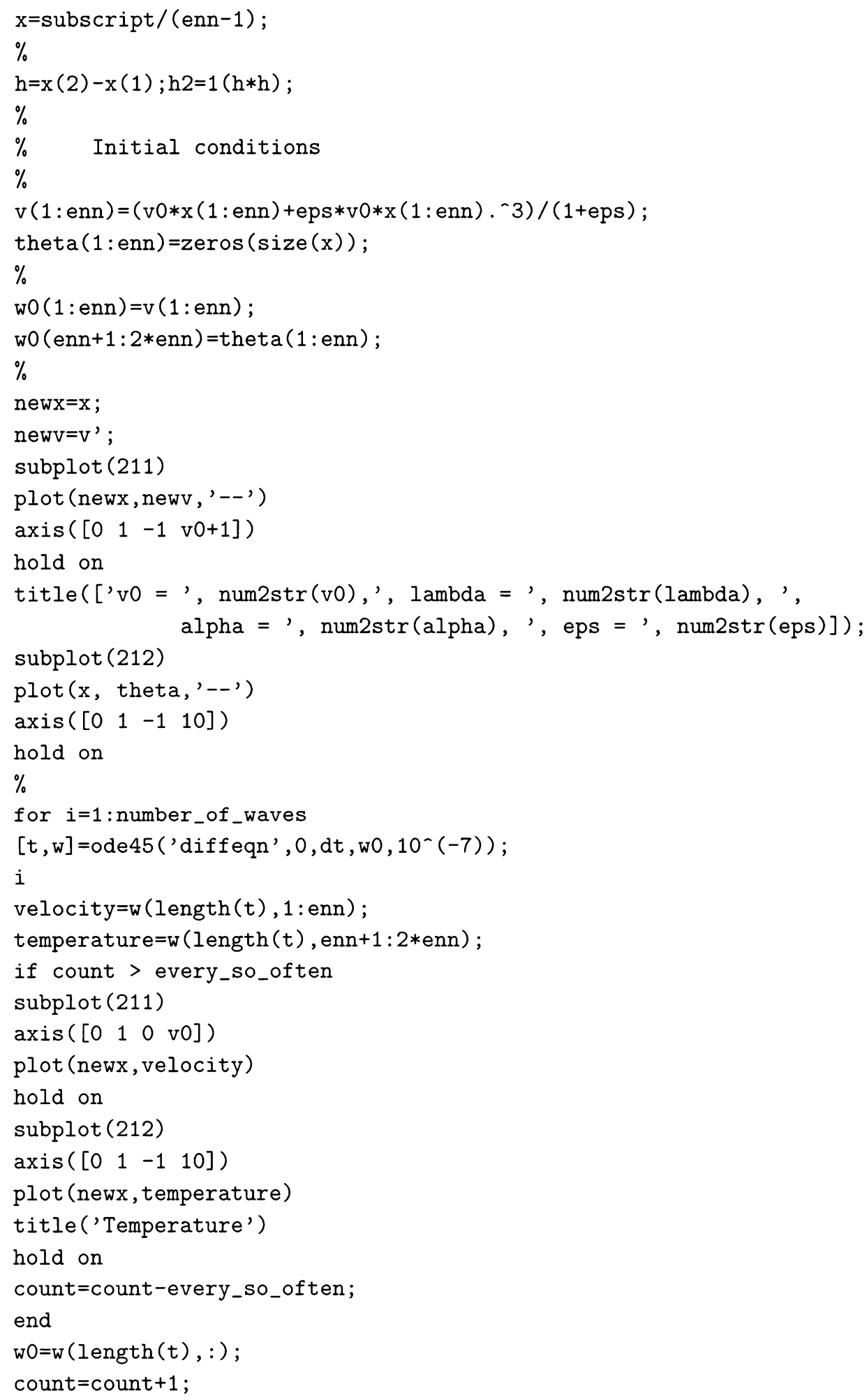


end

hold off

The above program uses the following M-file (called diffeqn.m in the main program): function wprime $=\operatorname{diffeqn}(t, w)$

global lambda alpha v0 enn h2 kappa;

$\%$

$\mathrm{v}(1: \mathrm{enn})=\mathrm{w}(1: \mathrm{enn})$

theta $(1: e n n)=w(e n n+1: 2 * e n n)$;

$\%$

$\operatorname{vr}(1: e n n-1)=\mathrm{v}(2: e n n) ; \operatorname{vr}(e n n)=\mathrm{v} 0 ;$

$\mathrm{v} 1(2: e n n)=\mathrm{v}(1: e n n-1) ; \mathrm{vl}(1)=0$;

$\operatorname{thetar}(1: e n n-1)=\operatorname{theta}(2: e n n) ; \operatorname{thetar}(e n n)=\operatorname{theta}(e n n-1) ;$

thetal $(2: e n n)=\operatorname{theta}(1: e n n-1) ; \operatorname{thetal}(1)=\operatorname{theta}(2) ;$

$\%$

term $=\exp (-$ alpha*theta) ;

vprime $=\mathrm{h} 2 *$ term. $*(\mathrm{vr}-2.0 * \mathrm{v}+\mathrm{vl})-0.25 * \mathrm{~h} 2 *$ alpha $*$ term. $*(\mathrm{vr}-\mathrm{vl})$.

*(thetar-thetal);

$\operatorname{vprime}(1)=0 ; \operatorname{vprime}(e n n)=0 ;$

thetaprime $=\mathrm{h} 2 *$ lambda $*($ thetar $-2.0 *$ theta + thetal $)+0.25 *$ kappa $*$ h $2 *$ term.

$*(\mathrm{vr}-\mathrm{vl}) . *(\mathrm{vr}-\mathrm{vl})$;

$\%$

wprime=[vprime thetaprime];

\section{REFERENCES}

[1] J. H. Maddocks and R. Malek-Madani, Steady-state shear bands in thermo plasticity - I. Vanishing yield stress, Internat. J. Solids Struct. 29, 2039-2061 (1992)

[2] M. Reiner, Rheology, Elasticity and Plasticity, Handbuch der Physik 6, Editor S. Flugge, 434-549 (1958)

[3] A. Tzavaras, Effect of thermal softening in shearing of strain-rate dependent materials, Archive for Rational Mechanics, vol. 99, 1987, pp. 349-374

[4] D. A. French and S. M. F. Garcia, Finite element approximation of an evolution problem modeling shear band formation, Comput. Methods Appl. Mech. Engrg., vol. 118, 1994, pp. 153-163

[5] S. Jiang, Asymptotic stability of shearing flows of incompressible thermoviscous fluids, Nonlinear Analysis, vol. 24, no. 8, 1995, pp. 1207--1220 